\title{
Two-species, 3D, MHD Simulation of Europa's Interaction with Jupiter's Magnetosphere
}

\author{
Yifan Liu, Andrew F. Nagy, Konstantin Kabin, \\ Michael R. Combi, Darren L. DeZeeuw, Tamas I. Gombosi \\ Space Physics Research Laboratory, Department of Atmospheric, Oceanic and Space Sciences, \\ University of Michigan, Ann Arbor, MI, 48109
}

Kenneth G. Powell

Aerospace Engineering Department, University of Michigan, Ann Arbor, MI, 48109

\begin{abstract}
The interaction of Europa with the Jovian magnetosphere has been studied by using a two species ideal magnetohydrodynamic (MHD) numerical model. This model considers the upstream plasma in the Jovian magnetosphere and the molecular oxygen ions in the ionosphere of Europa, separately. We present results from simulation studies, which take into account impact ionization, recombination, and the effect of a possible induced dipole magnetic field of Europa. The total mass loading of the magnetospheric flow and the ionization frequency used in the model are consistent with the estimates of Europa's ionosphere and atmosphere. The multi-species MHD equations are solved by using a finite volume, high-order, Godunov-type method on an adaptively refined unstructured grid, which allows detailed modeling of the region near Europa's surface, while still resolving both the upstream region and the satellite's wake. We have paid special attention to the wake of Europa, in order to be able to make comparisons with the Galileo's E4 flyby observations, as well as other model calculations. The calculated escape flux of $\mathrm{O}_{2}^{+}$down the tail was found to be about $5.6 \times 10^{25} \mathrm{~s}^{-1}$.
\end{abstract}

\section{Introduction}

Jupiter's satellite Europa is located deep inside the Jovian magnetosphere. Surface gas densities of the order of $10^{7} \mathrm{~cm}^{-3}$ have been indirectly deduced [Hall et al., 1995; Kliore et al., 1997] and assumed to consist mostly of $\mathrm{O}_{2}$. Solar photoionization and particle impact ionization produce an ionosphere around $\mathrm{Eu}$ ropa. Electron impact ionization has been suggested to be the most dominant process producing new ions [Kliore et al., 1997; Saur et al., 1998]. The radio occultation observations (E4 and E6 flybys) made by the Galileo spacecraft show the existence of a tenuous ionosphere around Europa, with a maximum surface electron density of approximately $10^{4} \mathrm{~cm}^{-3}$ and a plasma scale height of about $240-440 \mathrm{~km}$ [Kliore et al., 1997]. Magnetic field, electron and ion density, bulk velocity

Copyright 2000 by the American Geophysical Union.

Paper number 1999GL003734.

0094-8276/00/1999GL003734\$05.00 and ion temperature measurements were obtained during the various Galileo flybys of Europa [Gurnett et al., 1998; Paterson et al., 1999; Kivelson et al., 1997, 1999]. Saur et al. [1998] developed a model for Europa's magnetospheric interaction with emphasis on the neutral atmosphere. Kivelson et al. [1999] proposed to explain the magnetic signature of Europa by assuming a small induced dipole. Recently, Kabin et al. [1999] studied the global interaction process around Europa by using a single-species MHD model. Their results agree well with the magnetic field and electron density measurements obtained during the E4 flyby. However, in order to further elucidate the interactions between Europa's ionosphere and Jupiter's magnetosphere, a multispecies MHD approach was developed and results from that model are presented in this paper.

\section{Model and numerical method}

Multi-species MHD models have been used previously to study the solar wind interaction with the nonmagnetic planets Venus and Mars [Tanaka and $\mathrm{Mu}$ rawski, 1997; Liu et al., 1999]. However, while Europa's intrinsic magnetic field is believed to be small, the solar wind interaction with Venus and Mars and Europa's interaction with Jupiter's magnetosphere are different. The main difference is the result of the fact that the relative plasma flow velocity at Europa is supersonic but sub-Alfvénic, and thus no bow shock forms, while the interaction at Venus and Mars is both supersonic and super-Alfvénic, resulting in a bow shock. We solve the ideal coupled MHD equation set, consisting of two continuity, one momentum, one energy and the magnetic induction equations. The source vector, $\mathbf{Q}$, used in the calculations is:

$$
\mathbf{Q}=\left(\begin{array}{c}
0 \\
S-\alpha \rho_{2}^{2} \\
-\nu\left(\rho_{1}+\rho_{2}\right) \mathbf{u} \\
0 \\
-\nu\left(\frac{1}{2}\left(\rho_{1}+\rho_{2}\right) u^{2}+\frac{p}{\gamma-1}\right)
\end{array}\right)
$$

where $\rho_{1}$ and $\rho_{2}$ are the mass densities of the first species (the upstream magnetospheric plasma) and the 
second species (the ionospheric $\mathrm{O}_{2}^{+}$), respectively, $p$ is the total thermal pressure of the plasma, $\mathbf{u}$ is the plasma velocity, $\gamma=\frac{5}{3}$, and $S$ is the $\mathrm{O}_{2}^{+}$mass source rate from impact ionization, which is equal to the product of the electron impact ionization frequency and the neutral $\mathrm{O}_{2}$ density. The production and loss terms for the first species were neglected. Mass-loading from impact ionization was added on the ram side. We selected an electron impact ionization rate of $1.0 \times 10^{-6} \mathrm{~s}^{-1}$, close to the value suggested by Saur et al. [1998]. We also set, after trying a number of reasonable values, the neutral $\mathrm{O}_{2}$ density to be given by $2.85 \times 10^{7} \exp \left(-\frac{R-R_{E}}{175 \mathrm{~km}}\right) \mathrm{cm}^{-3}$ (where $R-R_{E}$ is the distance from the surface, and the radius of Europa, $R_{E}=1569 \mathrm{~km}$ ), which corresponds to a column density of $5 \times 10^{18} \mathrm{~m}^{-2}$, and which agrees with the estimate of Hall et al. [1995]. The normalized coefficient, $\alpha$, is directly related to the dissociative recombination rate, which was taken to be $8 \times 10^{-8} \mathrm{~cm}^{3} \mathrm{~s}^{-1}$ [Schunk and Nagy, 2000], and the value of $\nu$, the ionneutral momentum collision frequency, was selected to be $1.0 \times 10^{-9}\left[\mathrm{O}_{2}\right] \mathrm{s}^{-1}$. We found that the ion-neutral momentum transfer collisions do not have a significant effect on the magnetospheric flow past Europa.

A modified version of the BATS-R-US (Block Adaptive Tree Solar-wind Roe-type Upwind Scheme) has been developed to solve the two-species MHD equations. The BATS-R-US solution method is a highly scalable, massively parallel, block-adaptive mesh refinement algorithm developed for space physics applications that uses recent algorithmic advances in high-resolution upwind schemes. Details of the approach are described by Powell et al. [1999] and Groth et al. [1999].

The reference frame we use in this paper is a Cartesian reference system in which the $X$ axis is parallel to the expected undisturbed plasma velocity (corotation direction), the $Z$ axis is parallel to the Jovian spin axis, and the $Y$ axis completes the right-hand system. In the simulation the upstream plasma velocity was tilted $20^{\circ}$ with respect to the corotation direction, as suggested by Kabin et al. [1999]. The tilt of the upstream plasma velocity is implied by the density and energetic particle measurements during the E4 flyby [Kabin et al., 1999].

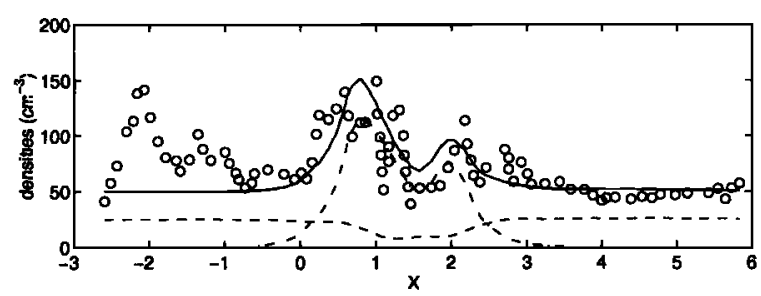

Figure 1. The calculated number densities $\left(n_{e}, n_{1}\right.$, and $n_{2}$ ) and the observed electron density along the E4 flyby. The solid, dashed and dash-dot lines denote the calculated electron $\left(n_{e}\right)$, upstream ion $\left(n_{1}\right)$, and $\mathrm{O}_{2}^{+}$ $\left(n_{2}\right)$ densities, respectively. The circles are the electron density data of Gurnett et al. [1998].
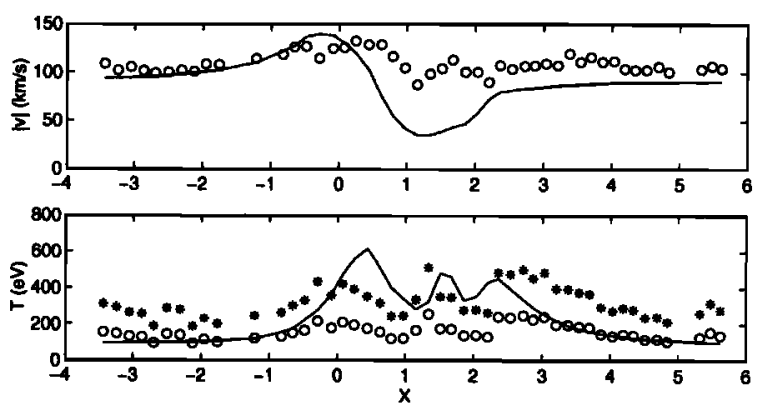

Figure 2. Speed and temperature profiles along the E4 flyby. The solid lines are the plasma speed (upper panel) and plasma temperature (lower panel) from the model results. The circles are the ion speed (upper panel) and ion temperature (lower panel) data of $\mathrm{Pa}$ terson et al. [1999]. The values marked by the stars are twice the measured ion temperatures.

The input values of the upstream parameters for our model were based on the Galileo magnetic field measurements [Kivelson et al., 1997] and on a model of the Io plasma torus [Smyth and Combi, 1988] derived from the Voyager 1 plasma data. The upstream parameters used were as follows: mean molecular mass $24 \mathrm{amu}$, average charge state 2, plasma temperature $90 \mathrm{eV}$, density $25 \mathrm{~cm}^{-3}$, velocity $90.3 \mathrm{~km} \mathrm{~s}^{-1}$, and a uniform tilted Jovian magnetic field of $(63,-165,-420) \mathrm{nT}$. These parameters correspond to a sonic Mach number of 3.7 and Alfvénic Mach number of 0.22 .

A computational domain, defined by $-192 R_{E} \leq x \leq$ $64 R_{E},-128 R_{E} \leq y, z \leq 128 R_{E}$, was used in the calculations and the inner boundary was at the surface of Europa. 543,488 computational cells, with length ranging from $49 \mathrm{~km}$ to $12552 \mathrm{~km}$, were used in the simulation. The inner boundary conditions were specified as follows: $\rho_{1}=0, \mathbf{B}=\mathbf{B}_{\infty}+\mathbf{B}_{d}$, where $\mathbf{B}_{d}$, the dipole field used in our simulation, is $(0,64,0) \mathrm{nT} R_{E}^{3}, \mathrm{v}=0, \rho_{2}$ was taken to be the chemical equilibrium value on the ram side, and equal to $0.01 \rho_{\infty}$ on the wake side, where the subscript ' $\infty$ ' means the upstream values. The plasma temperature at the inner boundary was assumed to be $5000 \mathrm{~K}$ and the pressure was set accordingly.

\section{Results}

Figure 1 shows the calculated number densities $\left(n_{e}\right.$, $n_{1}$, and $n_{2}$ ) and the observed electron density along the E4 flyby. The solid line denotes the calculated electron density $\left(n_{e}\right)$, the dashed line denotes the density of the ion species from the upstream magnetospheric plasma $\left(n_{1}\right)$, and the dash-dot line denotes the density of $\mathrm{O}_{2}^{+}$ $\left(n_{2}\right)$. We have assumed that the upstream plasma has an average charge state of 2 , and assumed charge neutrality for the plasma, therefore $n_{e}=2 n_{1}+n_{2}$ in our model. Circles are the electron density data of Gurnett et al. [1998]. The agreement with the electron density data from the plasma wave instrument is very good. 
The two peaks of the density in the wake region correspond to the closest approach and the center of the true corotation wake, respectively. We can see that in the wake region where the plasma density peaks, it consists mainly of $\mathrm{O}_{2}^{+}$. The minimum density between the two peaks occurs at the center of the shifted wake (c.f. Figure 1 and Figure 4). From Figure 1 we can easily see the mixing of the magnetospheric plasma and ionospheric plasma, which is due to the fact that the upstream dynamic pressure (approximately $8.2 \mathrm{nPa}$ ) slightly exceeds the peak ionospheric thermal pressure (approximately $8.0 \mathrm{nPa}$ ).

Figure 2 presents the speed and temperature of the plasma along the E4 flyby. The circles are the ion speed and ion temperature data of Paterson et al. [1999]. The measured parameter is the ion temperature, while the model calculated the plasma temperature. There is no good experimental or theoretical information regarding the electron temperatures in the wake region of Europa; however it is not unreasonable, for comparison purposes, to double the measured ion temperature (see star symbols in Figure 2) in order to make comparison with the calculated plasma temperature, which yields a qualitatively good agreement. Both the measured and calculated plasma speeds reach their maximum in the terminator region, possibly as a result of the magnetic tension due to the magnetic field slipping over Europa. The calculated speed values in the wake, obtained by both this model and that of Kabin et al. [1999], are lower than the measured values.

Figure 3 shows the comparison of the model results with the magnetometer measurements along the E4 flyby. We can see that the agreement is quite good. Our assumed dipole field is similar to the one proposed by Kivelson et al. [1999]. We found that the dipole field of Europa used in the simulation is essential for matching the observed $B_{x}$ and $B_{y}$ components, while the $B_{z}$ component is basically controlled by the mass-loading
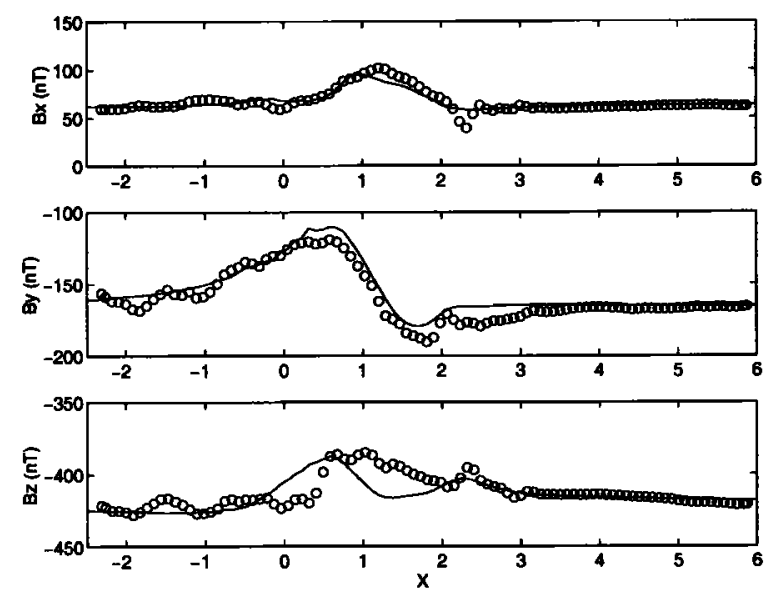

Figure 3. Magnetic field components along the E4 flyby. The solid lines are the results of the model. The circles are the data from magnetometer measurements.

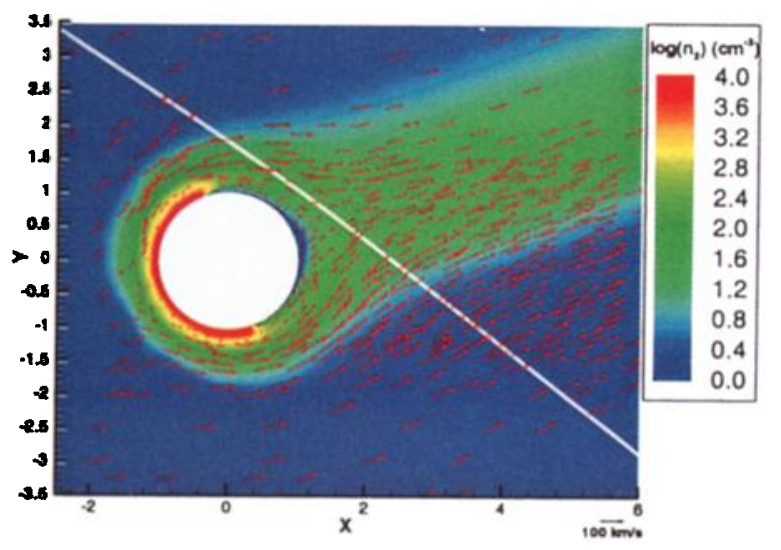

Figure 4. The density distribution of molecular oxygen ions in the ionosphere. The arrows show the velocity vectors. The white line shows the trajectory of Galileo's E4 flyby.

of the plasma, which is in agreement with Kabin et al. [1999].

Figure 4 shows the density distribution of the calculated molecular oxygen ions in the equatorial plane. The arrows show the velocity vectors. The observed peak densities were of the order of $10^{4} \mathrm{~cm}^{-3}$, near the surface [Kliore et al., 1997]. These six electron density profiles are insufficient to make definitive conclusions, concerning the variations with ram angle, although there maybe a slight indication that the ramside densities are the highest. The peak calculated $\mathrm{O}_{2}^{+}$density values are about $10^{4} \mathrm{~cm}^{-3}$ on the ram side, consistent with the observations. To estimate the amount of ionospheric plasma flow into the magnetosphere, we calculated the escape flux of molecular oxygen ions down the tail, which is approximately $5.6 \times 10^{25} \mathrm{~s}^{-1}$.

Figure 5 illustrate the magnetic field and field lines around Europa in the equatorial plane. A magnetic barrier, where the magnetic field piles up, formed in front of Europa, similar to those observed at Venus and Mars [Elphic et al., 1980; Acuña et al., 1998; Liu et al.,

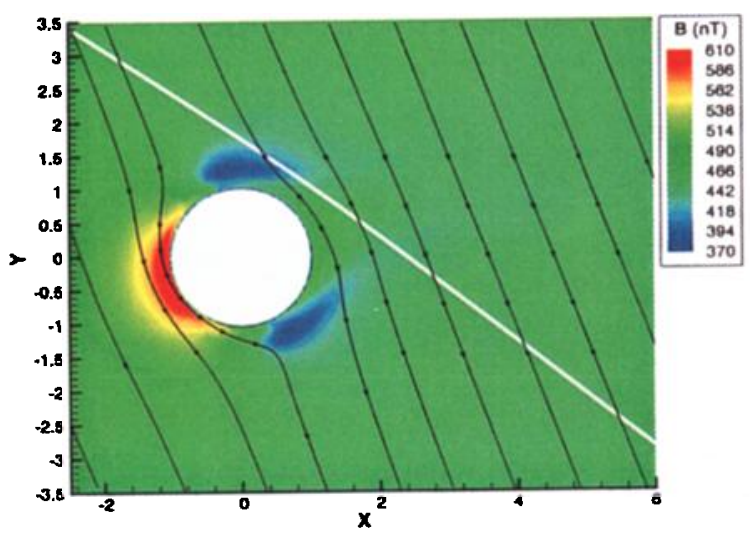

Figure 5. The magnetic field intensity around Europa. Lines with arrows are the magnetic field lines. The white line shows the trajectory of Galileo's E4 flyby. 
1999]. The tilt of the picture in the equatorial plane results from the rotation of the upstream plasma flow.

\section{Conclusion}

Here we presented the first results of our two species MHD model of the interaction of the Jovian magnetosphere with Europa. The two ions considered are the upstream ion species and $\mathrm{O}_{2}^{+}$, which is believed to be the major ion species in the ionosphere of Europa. The results have been compared with observed parameters, which have been obtained during Galileo's E4 flyby of December 19, 1996. The agreement between the model and observed density and magnetic field profiles is reasonably good. The major objective of the multi-species MHD model is to separate the ionospheric species from the magnetospheric species. The use of separate ionospheric and magnetospheric constituents allowed us to calculate the mass loading terms more accurately. However, the more important aspect of the two species approach is that it allows us to establish how the magnetospheric flow affects the ionospheric flow and structure and vice versa. For example we have been able to estimate the amount of ionospheric plasma flow into the magnetosphere. We also find that the presence of the ionosphere does create a "harder" obstacle to the magnetospheric flow, bending the magnetic field lines more and creating a stronger magnetic barrier.

Acknowledgments. This work was supported by NASA grants NAG5-4912, NAG5-8946 and HPCC CAN NCCS5146; additional computing resources on the GSFC HPCC Cray T3E (JSIMPSON) and NPACI Cray T3E at San Diego were also received and are acknowledged.

\section{References}

Acuña, M. H., et al., Magnetic field and plasma observations at Mars: Initial results of the Mars Global Surveyor mission, Science, 279, 1676, 1998.

Elphic, R. C., et al., Observations of the dayside ionopause and ionosphere of Venus, J. Geophys. Res., 85, 7679, 1980.

Groth, C. P. T., et al., A parallel solution-adaptive scheme for ideal magnetohydrodynamics, AIAA Paper 99-3273, June, 1999.
Gurnett, D. A., et al., Galileo plasma wave observations near Europa, Geophys. Res. Lett., 25, 237, 1998.

Hall, D. T., et al., Detection of an oxygen atmosphere on Jupiter's moon Europa, Nature, 373, 677, 1995.

Kabin, K., et al., On Europa's magnetospheric interaction: A MHD simulation of the E4 flyby, J. Geophys. Res., 104, $19,983,1999$.

Kivelson, M. G., et al., Europa's magnetic signature: Report from Galileo's pass on 19 December 1996, Science, 2\%6, $1239,1997$.

Kivelson, M. G., et al., Europa and Callisto: Induced or intrinsic fields in a periodically varying plasma environment, J. Geophys. Res., 104, 4609, 1999.

Kliore, A. J., et al., The ionosphere of Europa from Galileo radio occultations, Science, $277,355,1997$.

Liu, Y., et al., 3D multi-fluid MHD studies of the solar wind interaction with Mars, Geophys. Res. Lett., 26, 2689, 1999.

Paterson, W. R., L. A. Frank, and K. L. Ackerson, Galileo plasma observations at Europa: Ion energy spectra and moments, J. Geophys. Res., 104, 22,779, 1999.

Powell, K. G., et al., A solution-adaptive upwind scheme for ideal magnetohydrodynamics, J. Comp. Phys., 154, 284, 1999.

Saur, J., D. F. Strobel, and F. M. Neubauer, Interaction of the Jovian magnetosphere with Europa: Constraints on the neutral atmosphere, J. Geophys. Res., 103, 19,947, 1998.

Schunk, R. W., and A. F. Nagy, in Ionospheres, Cambridge University Press, in press, 2000.

Smyth, W. H., and M. R. Combi, A general model for Io's neutral gas clouds, II, Application to the sodium cloud, Astrophys. J.; 328, 888, 1988.

Tanaka, T., and K. Murawski, Three-dimensional MHD simulation of the solar wind interaction with the ionosphere of Venus: Results of two-component reacting plasma simulation, J. Geophys. Res., 102, 19,805, 1997.

M. R. Combi, D. L. DeZeeuw, T. I. Gombosi, K. Kabin, Y. Liu, A. F. Nagy, SPRL, Dept. of Atmospheric, Oceanic, and Space Sciences, U. of Michigan, Ann Arbor, MI 48109. (e-mail: liuyifan@engin.umich.edu) K. G. Powell, Aerospace Engineering Dept., U. of Michigan, Ann Arbor, MI 48109. (e-mail: powell@engin.umich.edu)

(Received December 14, 1999; revised February 23, 2000; accepted March 16, 2000.) 